\title{
An efficient quasi-3D particle tracking-based approach for transport through fractures with application to dynamic dispersion calculation
}

\author{
Submitted to Journal of Contaminant Hydrology \\ Lichun Wang ${ }^{1,2}$ and M. Bayani Cardenas ${ }^{1}$ \\ ${ }^{1}$ Department of Geological Sciences, University of Texas at Austin, Austin, Texas, USA \\ ${ }^{2}$ corresponding author: wanglichun@utexas.edu
}

\begin{abstract}
The quantitative study of transport through fractured media has continued for many decades, but has often been constrained by observational and computational challenges. Here, we developed an efficient quasi-3D random walk particle tracking (RWPT) algorithm to simulate solute transport through natural fractures based on a 2D flow field generated from the modified Local Cubic Law (MLCL). As a reference, we also modeled the actual breakthrough curves (BTCs) through direct simulations with the 3D advection-diffusion equation (ADE) and NavierStokes equations. The RWPT algorithm along with the MLCL accurately reproduced the actual BTCs calculated with the 3D ADE. The BTCs exhibited non-Fickian behavior, including early arrival and long tails. Using the spatial information of particle trajectories, we further analyzed the dynamic dispersion process through moment analysis. From this, asymptotic time scales were determined for solute dispersion to distinguish non-Fickian from Fickian regimes. This analysis illustrates the advantage and benefit of using an efficient combination of flow modeling and RWPT.




\section{Introduction}

Fractures may act as conduits for fluid flow and solute transport in fractured materials. Fracture roughness and tortuosity are the primary factors that control fluid flow and transport processes through fractures (Brown et al., 1995; Brush and Thomson, 2003; Tsang, 1984; Zheng et al., 2009; Zimmerman and Bodvarsson, 1996; Zimmerman et al., 1991). Thus, fundamental understanding and prediction of physical and chemical processes within single fractures is needed in order to analyze hydrological phenomena at various scales (Berkowitz, 2002). Despite its importance, the mathematical description of fluid flow and solute transport in single roughwalled fractures is typically simplified in most studies due to observational and computational limitations.

Fluid flow through rough-walled fractures is governed by the Navier-Stokes equations (NSE) and continuity equation, which are based on fluid momentum and mass conservation, respectively. For single-phase, incompressible, and steady fluid flow, the equations read as:

$$
\begin{array}{r}
\rho(\boldsymbol{u} \cdot \nabla) \boldsymbol{u}=-\nabla p+\mu \nabla^{2} \boldsymbol{u} \\
\nabla \cdot \boldsymbol{u}=0
\end{array}
$$

where $\rho$ is fluid density, $\boldsymbol{u}=[\mathrm{u}, \mathrm{v}, \mathrm{w}]$ is velocity vector, $p$ is total pressure, and $\mu$ is fluid dynamic viscosity. The resultant flow field from solving the NSE can thus be used to simulate solute transport dynamics.

Conservative solute transport through a fracture is described by the Eulerian advectiondiffusion equation (ADE):

$$
\partial C / \partial t+\boldsymbol{u} \nabla C=D_{m} \nabla^{2} C
$$


where $C$ refers to solute concentration and $D_{m}$ is the molecular diffusion coefficient. Although the $\mathrm{ADE}$ is the most accurate and complete formulation, solving it directly through rough-walled three-dimensional (3D) fractures remains challenging. This is because solving the ADE not only requires significant computational resources, but also suffers from numerical dispersion in the high-Peclet-number (Pe) regime (e.g., Patankar, 1980; Szymczak and Ladd, 2003). Reducing numerical dispersion requires refined discretization of the domain of interest, which increases the computational burden.

Recent studies solved the ADE using flow fields calculated from the NSE for twodimensional (2D) natural fractures (Cardenas et al., 2007; Cardenas et al., 2009; Wang and Cardenas, 2014). Using numerical models, the effect of roughness and inertial force on the solute transport was examined. However, only a few studies successfully implemented the NSE directly to digitized 3D fractures (Al-Yaarubi et al., 2005; Brush and Thomson, 2003; Wang et al., 2015), but none of those 3D cases considered the effect of the NSE-based flow field on solute transport; i.e., through solving the ADE directly. Further computational investigations of how roughness and inertial force affect solute transport within 3D fractures have been limited due to computational expense. To circumvent the difficulty of solving the ADE and associated NSE to simulate solute transport through 3D fractures, most researchers have used the Lagrangian random walk particle tracking algorithm (RWPT) with 2D flow fields generated from the classical Local Cubic Law (LCL) (e.g., Detwiler et al., 2000; James et al., 2005; Reimus, 1995; Zheng et al., 2009). This approach is popular because 2D simulations are more computationally convenient and efficient.

The LCL, sometimes called Reynolds equation, is a linearized or vertically-integrated version of the NSE widely used to characterize the flow field in rough-walled fractures (Brown 
et al., 1995; Zimmerman et al., 1991):

$$
\nabla \cdot\left(b(x, y)^{3} \nabla p\right)=0
$$

where $b$ is the local vertical aperture, which is the void space between the top and bottom fracture surfaces. The underlying assumptions that allow the classical LCL to be used in lieu of the NSE are: (1) inertial force is sufficiently low (Reynolds number $(R e)$ ranging $10^{-5}-10^{-1}$ ) such that the transmissivity of fractures is independent of Reynolds number (Al-Yaarubi et al., 2005; Zimmerman et al., 2004), and (2) the fracture mid-surface plane is smooth enough compared to the mean aperture such that the undulation of the mid-surface plane can be neglected. However, natural fractures may have highly heterogeneous aperture fields (Berkowitz, 2002) and the fracture mid-surface plane may be tortuous. In addition, inertial force is not negligible even at moderate $R e$ regimes where flow tends to be non-Darcian (Qian et al., 2011). Consequently, the classical LCL consistently overestimates flow rates (Al-Yaarubi et al., 2005; Brush and Thomson, 2003; Konzuk and Kueper, 2004; Mourzenko et al., 1995; Nicholl et al., 1999), and thus may misrepresent transport processes as well.

To compensate for the discrepancy in flow rates estimated through the LCL so it can more accurately be applied for solute transport modeling, Detwiler et al. (2002) proposed and calculated a flow field correction coefficient based on observations from physical flow experiments. However, without prior and sample-specific knowledge derived from experiments, the widespread application of the classical LCL remains questionable, and thus further investigations of transport process with flow fields derived from solving the LCL might lead to erroneous results.

The goal of this study is to develop an approach for accurate and efficient calculation of 
transport, and by necessity flow, through single fractures to better understand the character and nature of transport, particularly quantification of the dynamic dispersion coefficient to describe non-Fickian transport and its transition to Fickian transport, if these are present. To achieve this goal, we first calculated BTCs from the ADE based on a flow field resulting from numerically solving the NSE, the results of which are considered the real or actual flow and transport fields. Seeking an alternative to the computationally intensive solution of the ADE, we then developed an efficient quasi-3D RWPT that also produced BTCs, but this time based on flow fields modeled through the LCL and a recently developed modified Local Cubic Law (MLCL) (Wang et al., 2015) that allows and corrects for local tortuosity, roughness, and low inertial force. The approach is quasi-3D in that the mean (or vertically-integrated) flow field is calculated along the fracture plane whereas vertical flow variations across the fracture plane are indirectly considered. Using the RWPT results, we analyzed the solute transport behavior, which exhibited non-Fickian properties. The analysis quantified the effective dispersion coefficient over time using spatial moment analysis, direct theoretical calculation of the related transition time scales from nonFickian to Fickian transport regimes, and inverse modeling of the BTC through an effective 1D ADE.

\section{Computational modeling methods}

\subsection{Direct simulation of flow and transport through natural fractures}

\subsubsection{Flow field solution with the Navier-Stokes equations}

Here, we used single natural fractures studied previously by Cardenas et al (2007), and Wang et al. (2015) (referred to as H1, H2, and H3 in that study). The fractures are natural welded Santana tuff samples from the Trans-Pecos region of Texas, USA, and they were mapped through high-resolution X-ray computed tomography. Detailed fracture information was 
described by Wang et al. (2015).

Numerical modeling with the NSE and continuity equations (i.e., computational fluid dynamics (CFD) simulation) was implemented in COMSOL Multiphysics, a commercial finiteelement modeling software, where standard fluid properties for water were prescribed: $\rho=1000$ $\mathrm{kg} / \mathrm{m}^{3}$ and $\mu=1 \times 10^{-3} \mathrm{~Pa} \cdot \mathrm{s}$. To reduce numerical dispersion, finer elements were imposed around the boundary and coarser elements within the domain; Figure 1 in Wang et al. (2015) illustrates a tetrahedral finite-element mesh for one of the fractures (H3). The mean fluid flow in the longitudinal direction is driven by a specified pressure gradient. The fracture sides were set as no-slip boundary conditions. The steady-state CFD model took $2 \sim 3$ days to converge with the model needing up to $60 \mathrm{~Gb}$ of memory.

\subsubsection{Solute transport modeling with the advection-diffusion equation}

Solute transport through 3D fractures is described by the ADE (equation 2). In this study, $D_{m}=2.03 \times 10^{-9} \mathrm{~m}^{2} / \mathrm{s}$ for a typical conservative solute (Li and Gregory, 1974). Initial concentration in the fracture domain was:

$$
C=0 \quad 0<x<l, 0<y<w,-b<z<b, t=0
$$

where $l$ and $w$ are fracture length and width, respectively. The inlet was specified as a Dirichlet boundary, whereas the outlet was an open boundary:

$$
\begin{aligned}
& C=C_{0}=1 \quad x=0 \quad t \geq 0 \\
& \partial C / \partial n=0 \quad x=l \quad 0 \leq t \leq \infty
\end{aligned}
$$

where $n$ represents the normal direction to the outlet boundary. The solute transport model was also solved with COMSOL Multiphysics using the same mesh as the NSE with minimal numerical dispersion. The simulation was run up to $t=1$ week when the solute filled the entire domain; the model used up to $30 \mathrm{~Gb}$ of memory. Note that the effective Peclet number $P_{\text {eff }}<1$ 
such that numerical dispersion was negligible. The $P_{\text {eff }}=\langle u\rangle\langle b\rangle \mid D_{A D E}$, where $\langle u\rangle$ is the mean flow velocity, $\langle b\rangle$ is the arithmetic mean aperture, and $D_{A D E}$ is the fitted dispersion coefficient.

\subsection{Quasi-3D random walk particle tracking: algorithm and numerical implementation}

\subsubsection{Particle transport algorithm based on the modified LCL}

In this study, the same standard method for RWPT was implemented as used in numerous studies (e.g., Detwiler et al., 2000; James et al., 2005; James and Chrysikopoulos, 2001; Zheng et al., 2009) to study solute transport through natural fractures. However, we used the flow field derived from solving the MLCL (Wang et al., 2015) rather than by solving the LCL (as done by most previous studies), thus the variation of the mid-surface plane of aperture field (i.e. tortuosity), roughness and low inertial effects were considered. The MLCL reads as:

$$
\nabla \cdot\left[\frac{T_{x}}{C} \frac{\partial p}{\partial x} \cos \left(\emptyset_{x}\right) \vec{\imath}+\frac{T_{y}}{C} \frac{\partial p}{\partial y} \cos \left(\emptyset_{y}\right) \vec{\jmath}\right]=0
$$

where $T_{x}$ and $T_{y}$ are transmissivities in the $x$ and $y$ directions (both in the $x-y$ horizontal plane), $\phi_{x}$ and $\phi_{y}$ are flow orientation angles relative to horizontal (see Figure 2 in Wang et al. (2015) for graphical illustration), and $C$ is a correction factor for roughness and weak inertial effects (see Figure 5 in Wang et al. (2015) for an illustration of $C$ ). Equation (5) is a non-linear equation since $C$ is also a function of $p$ (or the pressure gradient); thus it is solved numerically using an iterative solver also implemented in COMSOL Multiphysics. Flow was driven by a mean pressure gradient between an inlet and an outlet face.

After the mean (or vertically-integrated) velocities were calculated based on the MLCL, particles were released at the inlet following a flux-weighted probability density distribution. Following the assumption that the flow field follows the mid-surface of the aperture field, i.e., the aperture field is symmetric about the mid-surface, the velocity in the $z$ direction (i.e., vertical) 
is thus negligible. Therefore, particle advection and diffusion in the $x$ and $y$ directions were specified as (James et al., 2005; Zheng et al., 2009):

$$
\begin{aligned}
& x^{n+1}=x^{n}+u\left(x^{n}, y^{n}, z^{n}\right) \Delta t+N(0,1) \sqrt{2 D_{m} \Delta t} \\
& y^{n+1}=y^{n}+v\left(x^{n}, y^{n}, z^{n}\right) \Delta t+N(0,1) \sqrt{2 D_{m} \Delta t}
\end{aligned}
$$

while only molecular diffusion occurs in the $z$-direction:

$$
z^{n+1}=z^{n}+N(0,1) \sqrt{2 D_{m} \Delta t} \quad(6-3)
$$

where $\left[x^{n}, y^{n}, z^{n}\right]$ is particle location in the Cartesian space at time $n \Delta t, n$ refers to the current time step, and $n+1$ is the succeeding time step, $N(0,1)$ is a random selection from the standard normal distribution, and $\left[u\left(x^{n}, y^{n}, z^{n}\right), v\left(x^{n}, y^{n}, z^{n}\right)\right]$ is the local 3D velocity field in the $x, y$ and $z$ directions. To minimize computational costs of using an explicitly modeled 3D velocity field (i.e., through the solution of the NSE), we assumed a parabolic velocity profile in the $z$ direction which is similar to previous studies (Detwiler et al., 2000; Detwiler et al., 2002; James et al., 2005; James and Chrysikopoulos, 2000; James and Chrysikopoulos, 2001; Zheng et al., 2009). That is, Poiseuille flow is assumed across the local aperture. Thus, the quasi-3D velocities in the $x$ and $y$ directions are:

$$
\begin{array}{ll}
u=\frac{3}{2} U\left\{1-4\left[\frac{z-z_{\text {mid }}}{b(x, y)}\right]^{2}\right\} & (7-1) \\
v=\frac{3}{2} V\left\{1-4\left[\frac{z-z_{\text {mid }}}{b(x, y)}\right]^{2}\right\} & (7-2)
\end{array}
$$

where $[U, V]$ are the 2D velocities in the $x$ and $y$ directions, respectively, calculated from solving the MLCL (Wang et al., 2015) and the classical LCL, $z_{\text {mid }}$ is the location of mid-surface of the 
aperture field in the $z$-direction. Hereafter, the velocity used for implementing the RWPT was solved through the MLCL, unless stated otherwise (e.g., when using a flow field modeled with the classical LCL).

The exclusion of advection in the $z$ direction requires modification because a particle travelling across cells with different apertures should adjust itself to the appropriate $z$ location. That is, a particle still moves up and down as it tracks the upward and downward movement of the mean flow (i.e., flow tortuosity). This formulation is implemented as:

$$
z^{n+1}=z_{m i d}^{n+1}+\frac{b^{n+1}}{b^{n}}\left(z^{n}-z_{m i d}^{n}\right)
$$

where $z^{n+1}$ and $z^{n}$ are the $z$ locations across different cells with different apertures $b^{n+1}$ and $b^{n}$, and associated mid-surfaces $z_{\text {mid }}{ }^{n+1}$ and $z_{\text {mid }}{ }^{n}$ at current and future time steps, respectively.

\subsubsection{Adaptive time stepping}

Solution accuracy and computational efficiency largely depend on the time step $\Delta t$. To improve solution accuracy, time steps need to be sufficiently small such that a moving particle experiences all of the fracture's local roughness, i.e., it does not jump over parts of the domain leading to aliasing. On the contrary, increasing $\Delta t$ speeds the computational execution of the transient transport problem. To strike a balance between these competing objectives, an adaptive time stepping approach that satisfies the condition that every advective distance is less than half the cell size was adopted, and diffusive distance is less than 1/20 of the local aperture. This method simultaneously ensures solution accuracy and computational efficiency (Detwiler et al., 2002; Reimus and James, 2002).

\subsubsection{Initial particle release and boundary conditions}


The resulting solution of the RWPT is sensitive to the particle release location and scheme. Ideally, the spatial distribution and number of particles introduced at the inlet should be proportional to the local fluid flux (James et al., 2005; Reimus, 1995). However, fluid flux varies horizontally ( $y$-direction) and vertically ( $z$-direction). To capture this variation, we first determined the number of particles within piecewise $y$ locations across the inlet $(x=0)$. That is, the probability distribution function (PDF) was constructed based on the flow contribution of each cell (i.e., fracture resolution), where the 2D flow rate was estimated from the MLCL (or LCL), and particles were assigned based on the PDF. Therefore, the prorated fraction of particles can be determined within associated inlet cells according to the PDF. Afterward, a random value following a uniform distribution between 0 and 1 was generated to specify the $y$ location of each particle within each cell. Lastly, $z$ location was determined similarly, but assuming that the local vertical velocity follows a parabolic profile, which was used for location weighting. More details on assignment of spatial distribution of individual particles can be found in the works of Reimus (1995) and James et al. (2005).

Because we will be comparing results from the RWPT to those from direct numerical simulation with the 3D ADE, we specified the top and bottom fracture surfaces and both side faces as no-flux boundaries. That is, we employed the reflection principle every time a particle hits walls to satisfy a no-flux condition without energy loss (Szymczak and Ladd, 2003).

\subsubsection{Implementation of random walk particle tracking}

The RWPT algorithm was implemented in MATLAB by releasing $N=10^{4}$ particles. Because each particle's movement is independent of other particles, we were able to use the Parallel Toolbox in MATLAB to keep track of 12 particles simultaneously during each computation; this further improved the computational efficiency. The RWPT was implemented 
on a high-performance computer with 16 shared CPUs, and it took $\sim 2$ wall clock days to finish and used up to $5 \mathrm{~Gb}$ of memory. Sensitivity analysis with varying number of particles showed that the numerical results were no longer impacted by the number of particles when $N=10^{4}$.

\subsection{Analysis of solute transport behavior}

\subsubsection{Breakthrough curves}

The cumulative fraction of particles reaching the outlet boundary over time represents the normalized concentration for the RWPT $\left(C^{\prime}{ }_{R W P T}\right)$, which is:

$$
C_{R W P T}^{\prime}(t)=\frac{N_{c}(t)}{N}
$$

where $N_{c}(t)$ is the time series of accumulated number of particle arrivals at the outlet. The $C^{\prime}{ }_{R W P T}$ represents the BTC resulting from the RWPT. BTCs were generated with RWPT for two 2D velocity fields: using both the LCL and MLCL.

The concentration resulting from solution of (2) and (4) can be used to estimate the fluxweighted BTC from the ADE (Wang and Cardenas, 2014):

$$
C_{A D E}(t)=\frac{\iint u C(t) d A}{\iint u d A}
$$

where $u$ is the longitudinal velocity, $C_{A D E}$ is the solute concentration, and $A$ is the outlet area. We then normalized the flux-weighted concentration for the $\operatorname{ADE}\left(C^{\prime}{ }_{A D E}\right)$ and time $\left(t^{\prime}\right.$, or pore volume) following:

$$
\begin{aligned}
C_{A D E}^{\prime}(t) & =\frac{C_{A D E}(t)}{C_{0}} \quad(11-1) \\
t^{\prime} & =\frac{Q t}{V} \quad(11-2)
\end{aligned}
$$


where $Q$ is the volumetric flux from solving the NSE, and $V$ is the fracture volume. The BTCs were used to analyze transport behavior.

\subsubsection{Spatial moment analysis for effective dispersion coefficient}

Transport processes may be characterized by the first and second spatial moments of the plume and/or particles. In this study, longitudinal moment analysis was used to quantify the effective dispersion coefficient (degree of spreading) by analyzing the spatial pattern of the particles over time. The first $\left(m_{1}\right)$ and second $\left(m_{2}\right)$ moments are:

$$
\begin{aligned}
& m_{1}(t)=\frac{1}{N} \int_{0}^{L} x(t) d x \\
& m_{2}(t)=\frac{1}{N} \int_{0}^{L} x(t)^{2} d x
\end{aligned}
$$

where $x(t)$ represents location of an individual particle in the $x$ direction. $m_{1}(t)$ is the longitudinal center of mass (or position) of a plume, increasing with time. The longitudinal spatial variance $\left(\sigma^{2}\right)$ of the plume represents the spreading distance around the center of mass of the plume, which is:

$$
\sigma(t)^{2}=m_{2}(t)-m_{1}(t)^{2}
$$

From $\sigma^{2}$, the dynamic effective dispersion coefficient $(D(t))$ can be estimated as:

$$
D(t)=\frac{\sigma(t)^{2}}{2 t}
$$

\subsubsection{Analysis of asymptotic time scale}

Non-Fickian transport is due to the mixing and spreading process failing to reach an asymptote when the time or where the length over which transport occurs are not large compared 
to the scale of variation of the velocity field (Koch and Brady, 1987). That is, under certain circumstances, e.g., when the velocity field is statistically stationary, $D$ within the non-Fickian regime increases asymptotically to the Fickian dispersion regime after a sufficient time and/or length scale has been exceeded. Therefore, the quantification of a dynamic $D$ can also be used to determine the asymptotic threshold time and length scales that separate non-Fickian from Fickian transport (Wang et al., 2012).

For solute transport through parallel plates with smooth and uniform surfaces, the asymptotic time and length scales can be theoretically calculated (Wang et al, 2012). For natural fractures with roughness and tortuosity, we replace the uniform aperture with the arithmetic mean aperture $(\langle b\rangle)$ to estimate the theoretical asymptotic time scale as:

$$
T=<b>^{2} /\left(4 D_{m}\right)
$$

Moreover, the estimated dynamic $D$ based on accurate particle trajectories from (14) can be used to quantify the actual asymptotic time scale (Table 1), such that non-Fickian and Fickian transport regimes can be separated.

\section{Results and discussion}

\subsection{Solute transport dynamics}

The solute transport dynamics are affected by preferential high-velocity zones (Figures 1 and 2). The RWPT with the MLCL captured the effects of preferential transport along the high velocity zones, with large-correlation-range effects resulting in fast breakthrough. Correlated low-velocity zones retarded the solute propagation process, and are the cause for the long tails in the BTCs.

Overall, the solute propagation front from RWPT at different times generally resembles 
that from the 3D ADE for all studied fractures (Figure 1). This illustrates the robustness of our RWPT approach using a quasi-3D velocity field to simulate solute transport. However, the spatial distribution of particles adds noise that is not observed in the solute concentration field from the 3D ADE; this is because a minority of particles reside in places where concentration is too low to accurately assess. In general, the RWPT with the MLCL flow field is capable of capturing the propagation front of the solute (Figure 1).

\subsection{Breakthrough curves showing non-Fickian behavior}

For reference, the BTCs derived from solving the 3D ADE are considered the true BTCs. The BTCs from the RWPT based on the MLCL are able to replicate the actual BTCs, but the LCL-based BTCs fail to do so (Figure 3a) and resulted in excessively early breakthrough.

The BTCs predicted by the ADE and RWPT with the MLCL are characteristic of early arrival and long tailing - the typical non-Fickian features. The early arrival is clearly demonstrated by the BTCs (Figure 3a), with the elapsed time for $C / C_{0}=0.5<1$ pore volume. Moreover, it takes at least 2.5 pore volumes for the solutes to completely fill up the entire domain, and for all the particles to reach the outlet boundary. This feature signifies the long tails of BTCs. Fundamentally, the non-Fickian transport is caused by velocity variation resulting from the fracture roughness (Wang and Cardenas, 2014).

However, the RWPT with the MLCL, while it generally captures the actual BTCs better than the LCL, might still miss some of the late tail as shown in one of the natural fractures (H3) (Figure 3a). The slight difference could be due to the shortcomings of the quasi-3D velocity field in representing the real 3D velocity field, which may have non-parabolic local velocity profiles or even reversed fluid flow (e.g., large eddies).

\subsection{Validity of the random walk particle tracking algorithm}


Previous studies have shown that transport simulation based on the modified flow field using the LCL through RWPT leads to underestimation of longitudinal $D$ by $12-19 \%$ compared to experimental results (Detwiler et al., 2000; Nicholl and Detwiler, 2001). The discrepancy could be due to intrinsic limitations of extending the local vertical velocity variation parabolically across the fracture aperture to mimic the actual 3D velocity field, or due to assumptions in the LCL, which disregards velocity variations that result from fracture roughness (Brown et al., 1995; Brush and Thomson, 2003; Mourzenko et al., 1995) and tortuosity (Tsang, 1984). In addition, the apparent shortcoming of the RWPT with the LCL could be due to aspects of the RWPT algorithm, e.g., how boundary conditions are implemented or how and where particles are released. Moreover, without correcting for the flow field to match experimental results (Detwiler et al., 2000), the RWPT would clearly overestimate transport velocity (Figure 3a).

In spite of the failure of RWPT with LCL, our results show that the RWPT with 2D velocity from the MLCL reproduces the overall transport process well. We show that not only does the RWPT capture the solute propagation front (Figure 1), but that the RWPT-MLCL reproduce the actual BTCs fairly well (Figure 3a). This further implies that the MLCL adequately represented the local vertically-integrated flow rate and can be useful for transport prediction. Based on the accurate particle trajectories, we illustrate how we can further estimate the dynamic $D$ over time.

\subsection{Quantification of dynamic dispersion coefficient and asymptotic time scale}

The dynamic $D$ based on (14) increases with time (Figure $3 b$ ) to its asymptotic value (i.e., Taylor dispersion coefficient; see Table 1). The asymptotic $D$ are fairly consistent with values derived from fitting a classical one-dimensional (1D) effective ADE to the BTCs (Table 1). 
Again, in terms of reproducing transport process through rough-walled 3D fractures, similar values of $D$ further verify the validity of the RWPT approach with velocity derived from solving the MLCL. The dynamic $D$ can be further used to quantify the asymptotic time scale (Figure $3 b$ ).

The theoretically-derived values of $T$ from (15) are shorter than the actual time when dynamic $D$ has reached its asymptotic value (Table 1). The difference can be partially attributed to the fracture roughness, defined as $\sigma_{b} /<\mathrm{b}>$ where $\sigma_{b}$ is the standard deviation in $b$, which extends the asymptotic travel time compared to that predicted by a parallel plates model (Wang and Cardenas, 2014). However, the roughness for H1 (0.39) and H3 (0.40) are similar, which is greater than $\mathrm{H} 2$ (0.31). The difference in $L$ with respect to $T$ is trivial for $\mathrm{H} 3$, while it is significant for $\mathrm{H} 1$. This is because other factors also play an important role in transport processes. Establishing the contributions from various factors should be a topic of future studies.

\section{Summary and conclusion}

An efficient and accurate quasi-3D random walk particle tracking algorithm (RWPT) that uses a vertically-integrated 2D velocity field calculated by solving the modified Local Cubic Law (MLCL) through natural fractures is presented in this study. In addition, we simulated the actual transport process by directly solving the advection-diffusion equation (ADE). The RWPT with MLCL reproduces the actual solute propagation front and breakthrough curves. This supports the robustness of RWPT in capturing transport processes, i.e., observed non-Fickian behavior. However and as expected, the RWPT with the classical LCL overestimates the mean velocity. Using the particle tracking results, we determined the dynamic effective dispersion coefficient from the first and second spatial moments of particle location. Following this, we determined the actual asymptotic scales to distinguish the Fickian from non-Fickian regimes for rough-walled fractures. This illustrates that such analysis is possible with a robust and efficient 
approach to transport modeling. Because of the smaller computational demand relative to direct simulations, the RWPT with the MLCL has potential for application to large, 3D fracture domains, which in turn allows for more comprehensive analysis of transport processes.

\section{Acknowledgements}

This work was supported as part of the Center for Frontiers of Subsurface Energy Security (CFSES) at the University of Texas at Austin, an Energy Frontier Research Center funded by the U.S. Department of Energy, Office of Science, Office of Basic Energy Sciences under Award Number DE-SC0001114. Additional support was provided by the Geology Foundation of the University of Texas. We thank the three anonymous reviewers for their constructive and useful comments.

\section{References}

Al-Yaarubi, A.H., Pain, C.C., Grattoni, C.A. and Zimmerman, R.W., 2005. Navier-stokes simulations of fluid flow through a rock fracture, Dynamics of Fluids and Transport in Fractured Rock vol. 162. Geophys. Monogr. Ser., pp. 55-64. AGU, Washington, DC.

Berkowitz, B., 2002. Characterizing flow and transport in fractured geological media: A review. Adv. Water Resour. 25(8-12): 861-884.

Brown, S.R., Stockman, H.W. and Reeves, S.J., 1995. Applicability of the Reynolds Equation for modeling fluid flow between rough surfaces. Geophys. Res. Lett. 22(18): 2537-2540.

Brush, D.J. and Thomson, N.R., 2003. Fluid flow in synthetic rough-walled fractures: NavierStokes, Stokes, and local cubic law simulations. Water Resour. Res. 39(4): 1085.

Cardenas, M.B., Slottke, D.T., Ketcham, R.A. and Sharp, J.M., Jr., 2007. Navier-Stokes flow and transport simulations using real fractures shows heavy tailing due to eddies. Geophys. Res. Lett. 34(14): L14404. 
Cardenas, M.B., Slottke, D.T., Ketcham, R.A. and Sharp, J.M., Jr., 2009. Effects of inertia and directionality on flow and transport in a rough asymmetric fracture. J. Geophys. Res. 114(B6): B06204.

Detwiler, R.L., Rajaram, H. and Glass, R.J., 2000. Solute transport in variable-aperture fractures: An investigation of the relative importance of Taylor dispersion and macrodispersion. Water Resour. Res. 36(7): 1611-1625.

Detwiler, R.L., Rajaram, H. and Glass, R.J., 2002. Experimental and simulated solute transport in a partially-saturated, variable-aperture fracture. Geophys. Res. Lett. 29(8): 1131-1134.

James, S.C., Bilezikjian, T. and Chrysikopoulos, C., 2005. Contaminant transport in a fracture with spatially variable aperture in the presence of monodisperse and polydisperse colloids. Stoch. Environ. Res. Ris. Assess. 19(4): 266-279.

James, S.C. and Chrysikopoulos, C.V., 2000. Transport of polydisperse colloids in a saturated fracture with spatially variable aperture. Water Resour. Res. 36(6): 1457-1465.

James, S.C. and Chrysikopoulos, C.V., 2001. An efficient particle tracking equation with specified spatial step for the solution of the diffusion equation. Chem. Eng. Sci. 56(23): 6535-6543.

Koch, D.L. and Brady, J.F., 1987. A non-local description of advection-diffusion with application to dispersion in porous media. J. Fluid. Mech. 180: 387-403.

Konzuk, J.S. and Kueper, B.H., 2004. Evaluation of cubic law based models describing singlephase flow through a rough-walled fracture. Water Resour. Res. 40(2): W02402.

Li, Y.-H. and Gregory, S., 1974. Diffusion of ions in sea water and in deep-sea sediments. Geochim. Cosmochim. AC. 38(5): 703-714. 
Mourzenko, V., Thovert, J.-F. and Adler, P., 1995. Permeability of a Single Fracture; Validity of the Reynolds Equation. Journal de Physique II 5(3): 465-482.

Nicholl, M.J. and Detwiler, R.L., 2001. Simulation of flow and transport in a single fracture: Macroscopic effects of underestimating local head loss. Geophys. Res. Lett. 28(23): $4355-4358$.

Nicholl, M.J., Rajaram, H., Glass, R.J. and Detwiler, R., 1999. Saturated flow in a single fracture: Evaluation of the Reynolds equation in measured aperture fields. Water Resour. Res. 35(11): 3361-3373.

Patankar, S.V., 1980. Numerical heat transfer and fluid flow. McGraw-Hill, New York.

Qian, J., Chen, Z., Zhan, H. and Luo, S., 2011. Solute transport in a filled single fracture under non-Darcian flow. Int. J. Rock Mech. Min. Sci. 48(1): 132-140.

Reimus, P. W. and James, S. C., 2002. Determining the random time step in a constant spatial step particle tracking algorithm. Chem. Eng. Sci. 57(21): 4429-4434.

Reimus, P. W. 1995. The use of synthetic colloids in tracer transport experiments in saturated rock fractures, Thesis, University of New Mexico, Albuquerque, New Mexico.

Szymczak, P. and Ladd, A.J.C., 2003. Boundary conditions for stochastic solutions of the convection-diffusion equation. Phys. Rev. E 68(3): 036704.

Tsang, Y.W., 1984. The effect of tortuosity on fluid flow through a single fracture. Water Resour. Res. 20(9): 1209-1215.

Wang, L. and Cardenas, M.B., 2014. Non-Fickian transport through two-dimensional rough fractures: Assessment and prediction. Water Resour. Res. 50(2): 871-884. 
Wang, L., Cardenas, M.B., Slottke, D.T, Ketcham, R.A., Sharp, J.M., 2015. Modification of the Local Cubic Law of fracture flow for weak inertia, tortuosity and roughness. Water Resour. Res. (in press).

Wang, L., Cardenas, M.B., Deng, W. and Bennett, P.C., 2012. Theory for dynamic longitudinal dispersion in fractures and rivers with Poiseuille flow. Geophys. Res. Lett. 39(5): L05401.

Zheng, Q., Dickson, S. and Guo, Y., 2009. Influence of aperture field heterogeneity and anisotropy on dispersion regimes and dispersivity in single fractures. J. Geophys. Res. 114(B3): B03205.

Zimmerman, R.W., Al-Yaarubi, A., Pain, C.C. and Grattoni, C.A., 2004. Non-linear regimes of fluid flow in rock fractures. Int. J. Rock Mech. Min. Sci. 41, Supplement 1(0): 163-169.

Zimmerman, R.W. and Bodvarsson, G.S., 1996. Hydraulic conductivity of rock fractures. Transport Porous Med. 23(1): 1-30.

Zimmerman, R.W., Kumar, S. and Bodvarsson, G.S., 1991. Lubrication theory analysis of the permeability of rough-walled fractures. Int. J. Rock Mech. Min. Sci., Geomechanics Abstracts 28(4): 325-331.

\section{Table}

Table 1. Parameters for fitting breakthrough curves (BTCs) through the advection-dispersion equation (Inverse 1D Model). The BTCs were solved from direct simulation (i.e., advection diffusion equation and Navier-Stokes equations). $u$ and $D_{\text {asymptotic }}$ represent velocity and asymptotic dispersion coefficient, respectively. Asymptotic values of $D$ and time scales $(L)$ were observed from dynamic evolution of effective $D$ (Figure 3b). Additionally, the theoretical $T$ were estimated following equation (15). 


\begin{tabular}{c|c|c|c|c|c}
\multirow{2}{*}{$\begin{array}{c}\text { Natural } \\
\text { fractures }\end{array}$} & \multicolumn{2}{|c|}{ Inverse 1D Model } & \multicolumn{2}{c|}{ RWPT (MLCL) } & Theoretical \\
\cline { 2 - 6 } & $u$ & $D_{\text {asymptotic }}$ & $D_{\text {asymptotic }}$ & $L[\mathrm{~s}]$ & $T[\mathrm{~s}]$ \\
\hline H1 & $9.10 \times 10^{-5}$ & $6.70 \times 10^{-8}$ & $6.90 \times 10^{-8}$ & $\sim 8600$ & 1377.6 \\
\hline H2 & $6.95 \times 10^{-5}$ & $2.28 \times 10^{-7}$ & $1.83 \times 10^{-7}$ & $\sim 697$ & 275.6 \\
\hline H3 & $1.97 \times 10^{-5}$ & $9.95 \times 10^{-8}$ & $1.05 \times 10^{-7}$ & $\sim 617$ & 564.2
\end{tabular}

\section{Figure captions}

Figure 1. Propagation of solute transport through natural fractures $(\mathrm{H} 1, \mathrm{H} 2$, and $\mathrm{H} 3$ as studied by Wang et al. (2015)) over time for (a) $\sim 0.1$ pore volume, (b) $\sim 0.5$ pore volume, and (c) $\sim 1.0$ pore volume, as represented by plume (concentration) and particles from solving the advectiondiffusion equation (ADE) and random walk particle tracking (RWPT), respectively.

Figure 2. Spatial distribution of actual 3D and corresponding 2D vertically-averaged normalized velocity in the mean flow directions (as indicated by arrows) through natural fractures (referred to as $\mathrm{H} 1, \mathrm{H} 2$, and $\mathrm{H} 3$ studied by Wang et al. (2015)). The 3D velocity fields were calculated by solving the 3D Navier-Stokes equations and the 2D velocity fields were calculated by solving the modified Local Cubic Law (Wang et al., 2015).

Figure 3. (a) Breakthrough curves for 3D natural fractures $(\mathrm{H} 1, \mathrm{H} 2$, and $\mathrm{H} 3$ as studied by Wang et al. (2015)) simulated from direct solution of the advection-diffusion equation (ADE) and random walk particle tracking (RWPT) with velocities from the modified Local Cubic Law (MLCL) and classical Local Cubic Law (LCL), respectively. $C / C_{0}$ is the dimensionless concentration; pore volume is defined in equation (10-2). (b) Corresponding dynamic dispersion coefficients $(D)$ increases with time, where dashed red lines indicate the asymptotic time scales with corresponding asymptotic dispersion coefficients (values shown in Table 1). 
a) $0.1 \mathrm{PV} \mathrm{H} 1$
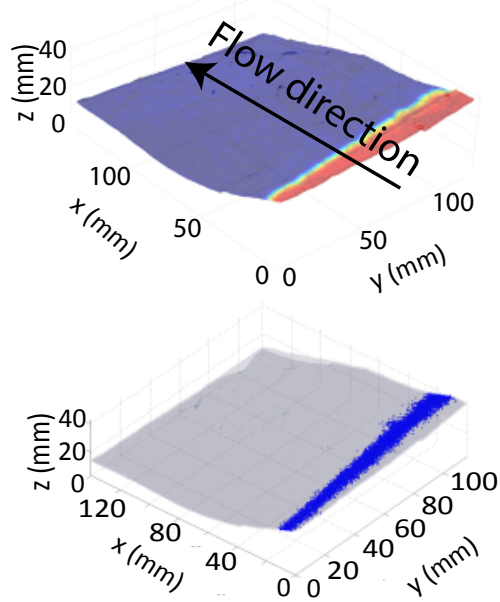

b) $0.5 \mathrm{PV}$
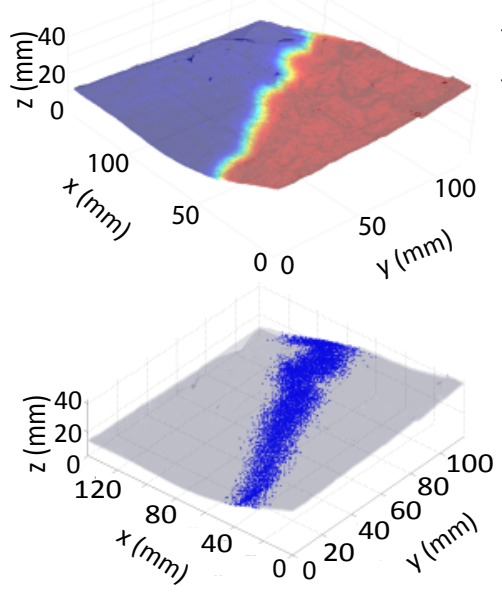

c) $\sim 1.0 \mathrm{PV}$
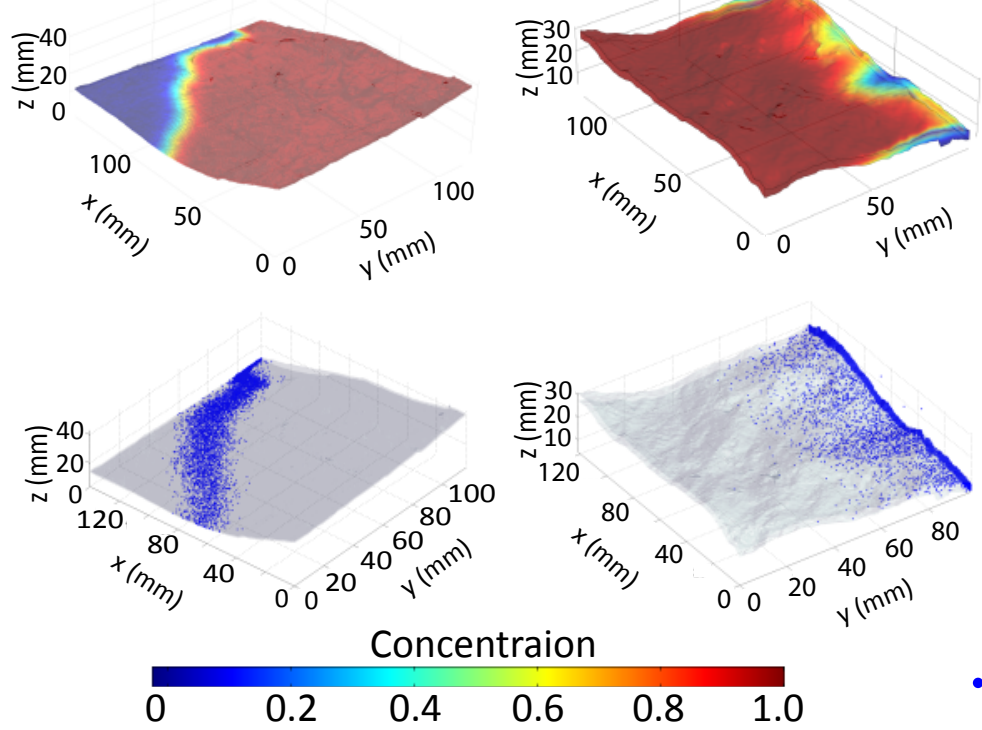

$\mathrm{H} 2$
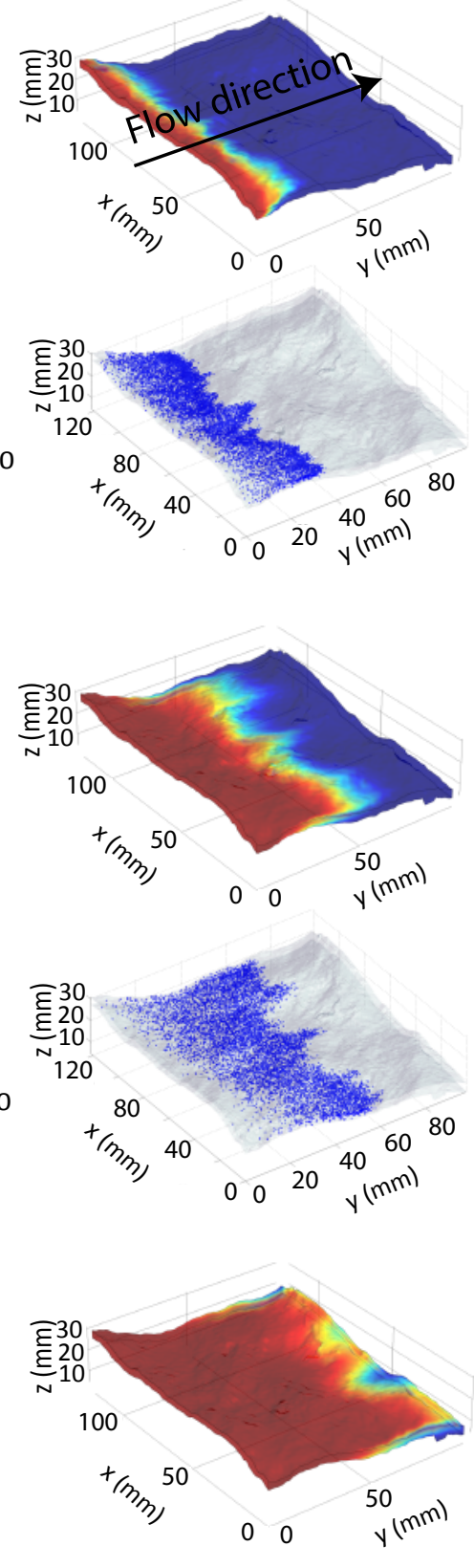
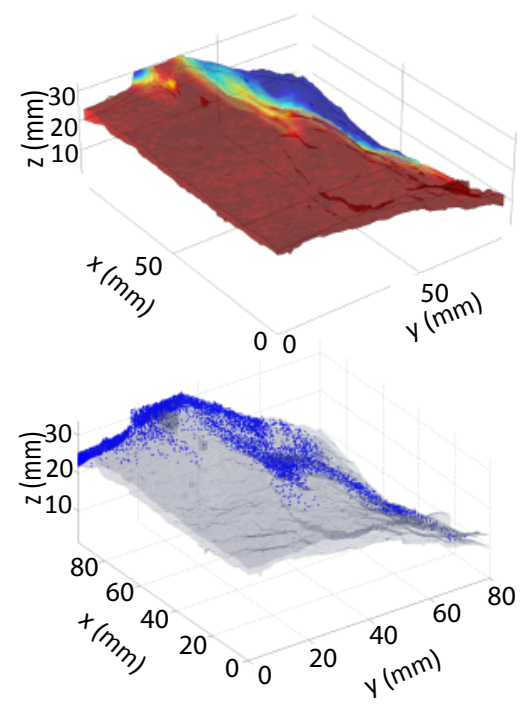

- Released particles
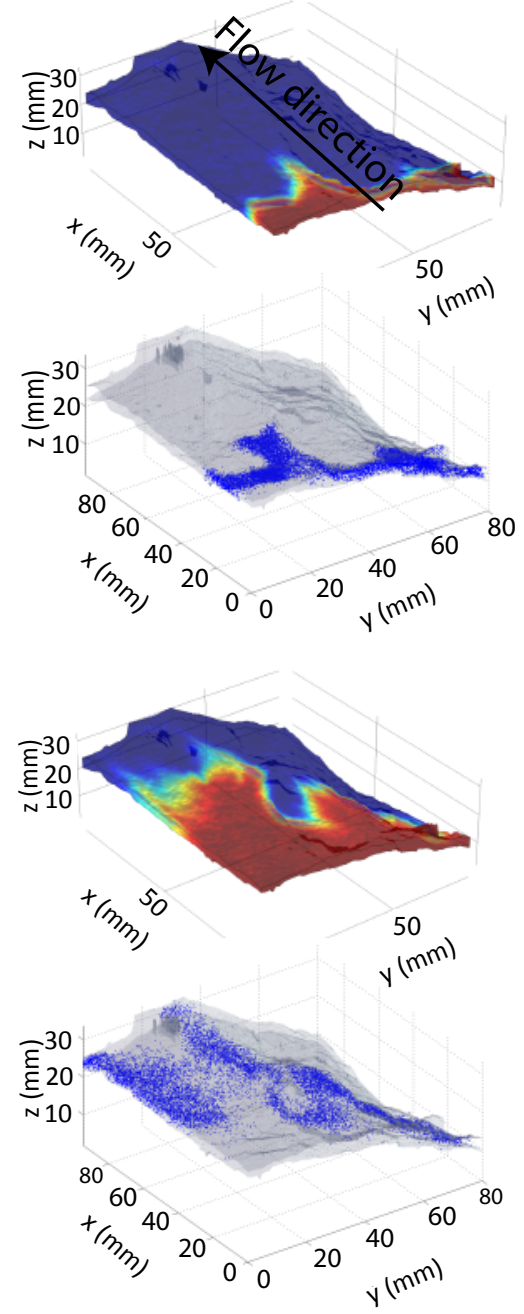

Figure 1. Propagation of solute transport through natural fractures $(\mathrm{H} 1, \mathrm{H} 2$, and $\mathrm{H} 3$ as studied by Wang
et al. (2015)) over time for (a) $\sim 0.1$ pore volume, (b) $\sim 0.5$ pore volume, and (c) $\sim 1.0$ pore volume, as represented by plume (concentration) and particles from solving the advection-diffusion equation (ADE) and random walk particle tracking (RWPT), respectively. 


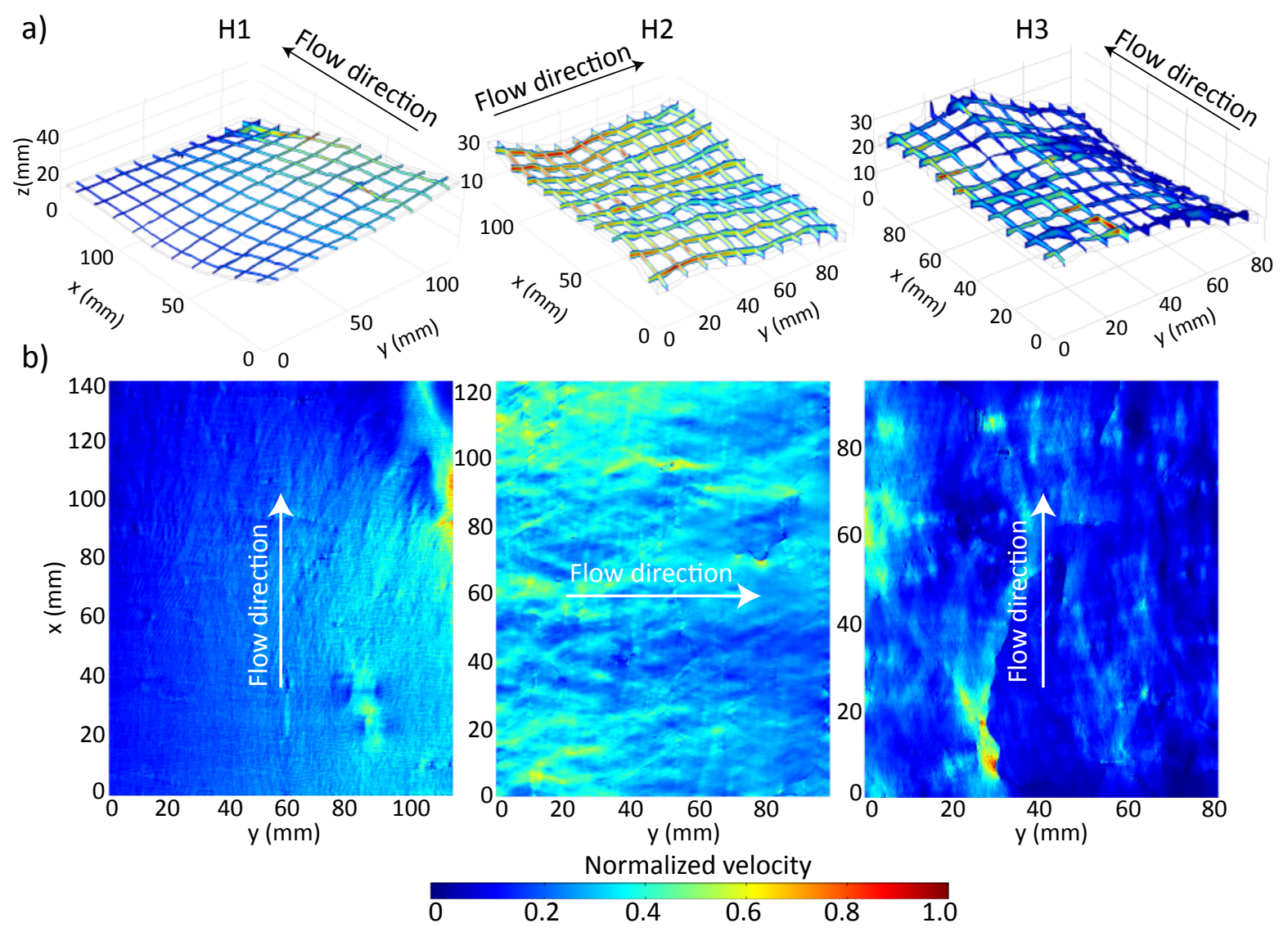

Figure 2. Spatial distribution of actual $3 \mathrm{D}$ and corresponding $2 \mathrm{D}$ vertically-averaged normalized velocity in the mean flow directions (as indicated by arrows) through natural fractures (referred to as H1, H2, and H3 studied by Wang et al. (2015)). The 3D velocity fields were calculated by solving the 3D Navier-Stokes equations and the 2D velocity fields were calculated by solving the modified Local Cubic Law (Wang et al., 2015). 
a) $\mathrm{H} 1$ $\mathrm{H} 2$ H3
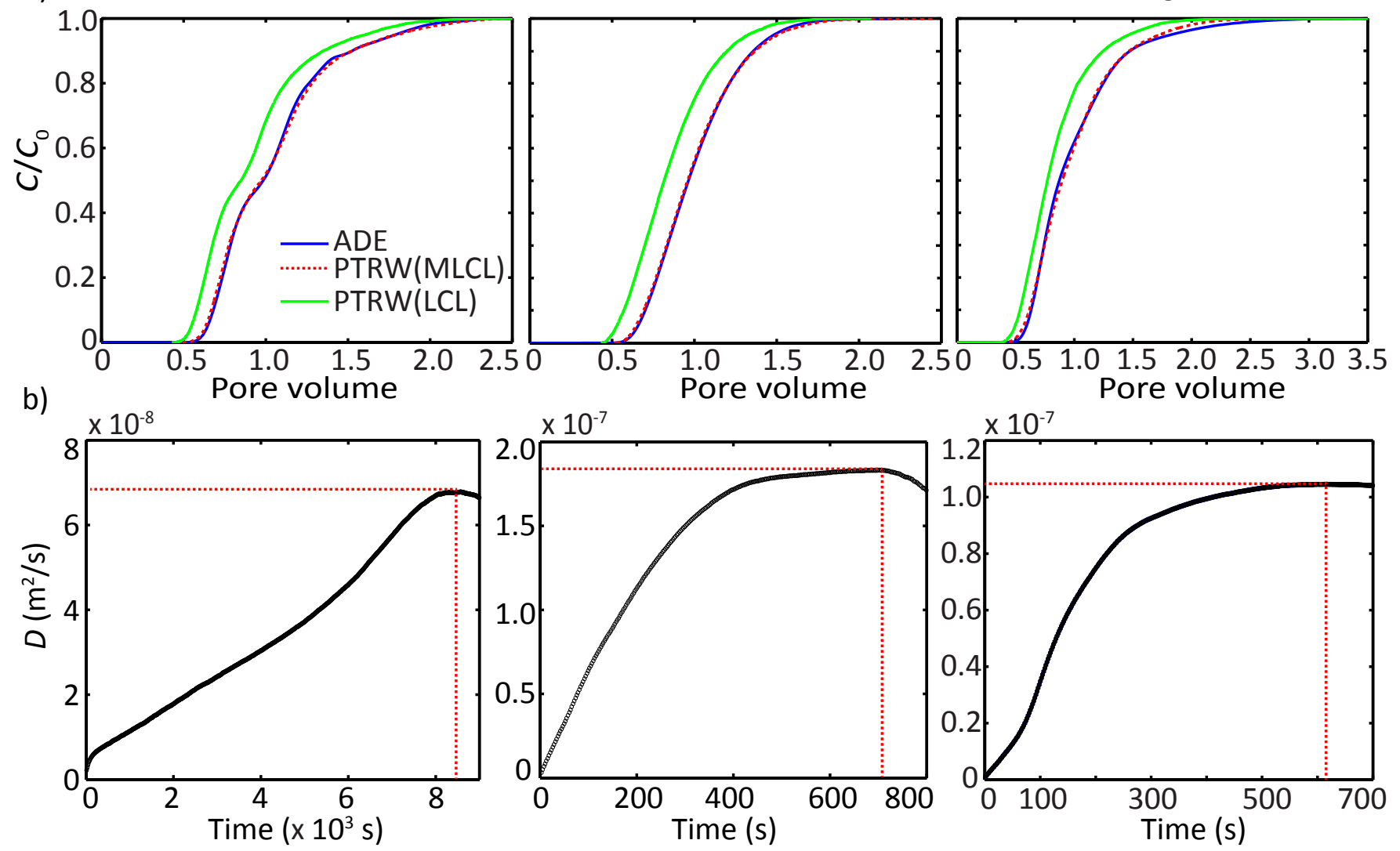

Figure 3. (a) Breakthrough curves for 3D natural fractures $(\mathrm{H} 1, \mathrm{H} 2$, and $\mathrm{H} 3$ as studied by Wang et al. (2015)) simulated from direct solution of the advection-diffusion equation (ADE) and random walk particle tracking (RWPT) with velocities from the modified Local Cubic Law (MLCL) and classical Local Cubic Law (LCL), respectively. $C / C_{0}$ is the dimensionless concentration, and pore volume is defined in equations $(10-2)$. (b) Corresponding dynamic dispersion coefficients $(D)$ increases with time, where dashed red lines indicate the asymptotic time scales with corresponding asymptotic dispersion coefficients (values shown in Table 1). 